\title{
Parametrical analysis of Friction Stir Welding of dissimilar aluminum AA7075 and AA2024 using Graph Labeling
}

\author{
J.Jayapriya ${ }^{1}$, D. Muruganandam ${ }^{2}$, K. Thirusangu ${ }^{3}$ and Sushil lal das ${ }^{4}$ \\ ${ }^{1,2}$ Research Scholar, Sathyabama University, Chennai-119 \\ ${ }^{3}$ Department of Mathematics, S.I. V.E.T College, Chennai-73 \\ ${ }^{4}$ Department of Mechanical Engineering, Jeppiaar Engineering College, Chennai-119 \\ priyanandam_1975@rediffmail.com, murudurai@gmail.com, kthirusangu@gmail.com
}

\begin{abstract}
In this paper the numerical data involved during the processes of welding is stored over a graph structure and its changes during compression are analyzed by using max/mini edge labeling.
\end{abstract}

Keywords: Graph, Function, Labeling, Alloy, Welding, Max/mini labeling.

AMS Subject Classification: $05 C 78$

\section{Introduction}

A graph labeling technique is an assignment of integers to the vertices or edges or both subject to certain conditions. Labeled graphs are becoming an increasingly useful family of Mathematical Models from a broad range of applications. The graph labeling problem that appears in graph theory has a fast development recently. This problem was first introduced by Alex Rosa in 1967. Since Rosa's article, many different types of graph labeling problems have been defined around this. This is not only due to its mathematical importance but also because of the wide range of the applications arising from this area, for instance, X-rays, crystallography, coding theory, radar, astronomy, circuit design, and design of good Radar Type Codes, Missile Guidance Codes and Convolution Codes with optimal autocorrelation properties and communication design. An enormous body of literature has grown around this subject is about 1300 papers. They gave birth to families of graphs with attractive names such as graceful, Harmonious, felicitous, elegant, cordial, magic, antimagic, bimagic and prime labeling etc. A useful survey to know about the numerous graph labeling methods is the one by Gallian (2011). All graphs considered here are finite simple, connected and undirected. Friction stir welding (FSW) is a solid state welding process for joining of alloys such as joining aluminum, magnesium and copper alloys and has been employed in several industrial application. The various parameters are such as rotational speed, longitudinal speed and axial force etc. The aim of this study is to investigate the effect of different rotational speed and tool pin profile on the weld quality of AA2024 aluminum which has gathered wide acceptance in the fabrication of light weight structures requiring a high strength-to-weight ratio. The appearance of the weld is found good and no obvious defect is found using these tools. The grain of the weld nugget is very fine and the precipitation distributes equally. Consequently, the obtained results elucidate the variation of stress as a function of strain and the effect of different rotational speed and pin profiles on yield strength and elongation.

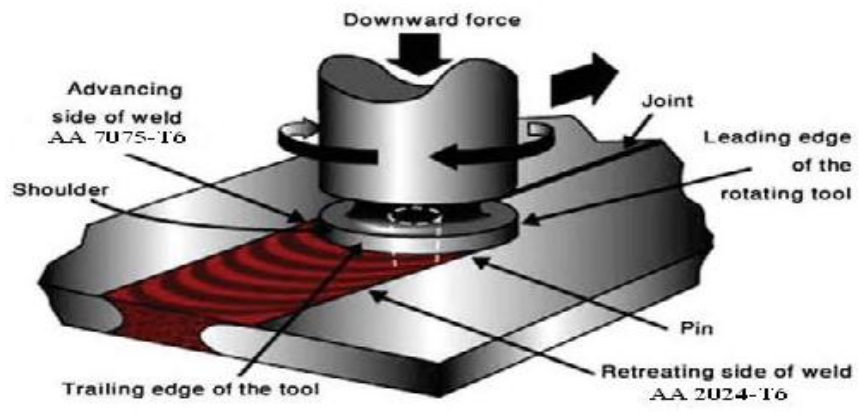

Fig 1.Showing the schematic diagram of FSW

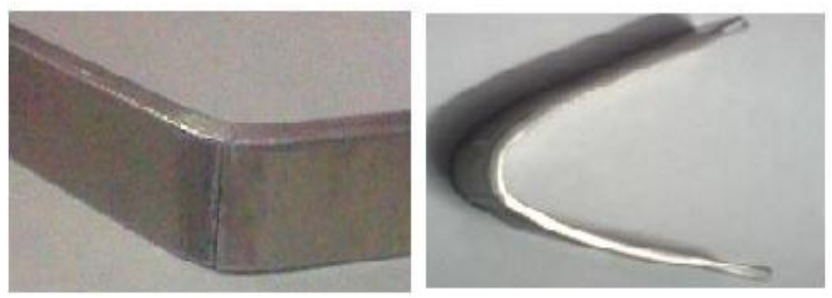

Fig 2. AA2024-AA7075 compressive tested specimens for Taper threaded tool (a) TT1 (b) TT2 and for Straight threaded tool (c) ST1 (d) ST2 
Table. 1 Typical parameter combinations used for investigation

\begin{tabular}{|l|c|c|c|c|}
\hline $\begin{array}{c}\text { Types of } \\
\text { Tool used }\end{array}$ & $\begin{array}{c}\text { Notation } \\
\text { used }\end{array}$ & $\begin{array}{c}\text { Rotational } \\
\text { speed } \\
\text { (rpm) }\end{array}$ & $\begin{array}{c}\text { Welding } \\
\text { speed } \\
(\mathrm{mm} / \mathrm{min})\end{array}$ & $\begin{array}{c}\text { Axial } \\
\text { load(KN) }\end{array}$ \\
\hline \multirow{4}{*}{$\begin{array}{c}\text { Taper } \\
\text { threaded }\end{array}$} & TT1 & 600 & 30 & 2.5 \\
\cline { 2 - 5 } & TT2 & 700 & 30 & 2.5 \\
\cline { 2 - 5 } & TT3 & 800 & 30 & 2.5 \\
\cline { 2 - 5 } & TT4 & 900 & 30 & 2.5 \\
\cline { 2 - 5 } & TT5 & 800 & 20 & 2.5 \\
\cline { 2 - 5 } & TT6 & 800 & 40 & 2.5 \\
\hline \multirow{5}{*}{$\begin{array}{l}\text { Straight } \\
\text { threaded }\end{array}$} & TT7 & 800 & 50 & 2.5 \\
\cline { 2 - 5 } & TT8 & 800 & 60 & 2.5 \\
\cline { 2 - 5 } & ST1 & 600 & 30 & 2.5 \\
\cline { 2 - 5 } & ST2 & 700 & 30 & 2.5 \\
\cline { 2 - 5 } & ST3 & 800 & 30 & 2.5 \\
\cline { 2 - 5 } & ST5 & 900 & 30 & 2.5 \\
\cline { 2 - 5 } & ST6 & 700 & 20 & 2.5 \\
\cline { 2 - 5 } & ST7 & 700 & 40 & 2.5 \\
\cline { 2 - 5 } & ST8 & 700 & 60 & 2.5 \\
\hline
\end{tabular}

Table 2. Compressive properties for typical combinations used for investigation

\begin{tabular}{|c|c|c|c|}
\hline Combinations & $\begin{array}{c}\text { Tool } \\
\text { rotation } \\
\text { speed }(\mathrm{rpm})\end{array}$ & $\begin{array}{c}\text { Root } \\
\text { bend }\end{array}$ & $\begin{array}{c}\text { Face } \\
\text { bend }\end{array}$ \\
\hline $\begin{array}{c}\text { AA2024 }- \\
\text { AA7075 (TT1) }\end{array}$ & 600 & $\begin{array}{c}\text { Crack } \\
\text { observed } \\
\text { after 42 }\end{array}$ & $\begin{array}{c}\text { No } \\
\text { cracks } \\
\text { observed }\end{array}$ \\
\hline
\end{tabular}

\section{Main Definition}

Max/ mini edge labeling: Let $G(V, E)$ be a simple undirected graph where $V=\left\{x_{1}, x_{2} \ldots x_{m}\right\}$ is a set of vertices or nodes, $E=\left\{e_{1}, e_{2} \ldots e_{n}\right\}$, a set of pairs of distinct vertices, called edges or lines. Let $f: V \rightarrow R^{*}$, where $R^{*}=R-\{0\}$ is a set of real numbers. If the weight of each edge is defined by $e_{k}=\frac{\max \left\{f\left(x_{i}\right), f\left(x_{j}\right)\right\}}{\min \left\{f\left(x_{i}\right), f\left(x_{j}\right)\right\}}, 1 \leq i, j \leq m, n$ and $\mathrm{i} \neq \mathrm{j}, \mathrm{k}=1,2,3, \ldots,|\mathrm{E}|$ is distinct then the labeling is called as Max/mini edge labeling. A graph which admits a Max/mini edge labeling is called as Max/ mini edge labeling graph.

\section{Analyzing Welding by using Graph Labeling}

Let $\mathrm{G}(\mathrm{V}, \mathrm{E})$ represent a graph whose vertices $\mathrm{V}$ are $V=\left\{\left(a_{i j}\right) ; 1 \leq i \leq m, 1 \leq j \leq n\right\}, m, n \in N$ where $N$ is a set of natural numbers and $|\mathrm{V}|=\mathrm{mn}$. (i.e. $\mathrm{a}_{\mathrm{ij}}$ represent the
Vol. 5 No. 8 (August 2012) ISSN: 0974- 6846

vertices identified by $i^{\text {th }}$ row and $j^{\text {th }}$ column, where $\mathrm{i}=1,2, \ldots, \mathrm{m}, \mathrm{j}=1,2, \ldots, \mathrm{n}$.) which gives a bijective mapping defined as follows

Case (i): When $\mathrm{n}$ is even the bijection $\mathrm{f}: \mathrm{V} \rightarrow \mathrm{R}^{*}$ is defined as the following

$$
f\left(a_{i j}\right)= \begin{cases}2 i-1 & j=1 ; 1 \leq i \leq m, \\ a_{m(j-1)}+2 i & 2 \leq j \leq n / 2 ; 1 \leq i \leq m \\ 2 i & j=n / 2+1 ; 1 \leq i \leq m \\ a_{m(-1)}+2 i & n / 2+2 \leq j \leq n ; 1 \leq i \leq m .\end{cases}
$$

Case (ii): When $\mathrm{n}$ is odd the bijection

$\mathrm{f}: \mathrm{V} \rightarrow \mathrm{R}^{*}$ is defined as the following

$$
f\left(a_{i j}\right)= \begin{cases}2 i-1 & j=1 ; 1 \leq i \leq m \\ a_{m(j-1)}+2 i & 2 \leq j \leq\lfloor n / 2 j ; 1 \leq i \leq m \\ 2 i & j=\lfloor n / 2\rfloor+1 ; 1 \leq i \leq m \\ a_{m(j-1)}+2 i & \lfloor n / 2\rfloor+2 \leq j \leq n ; 1 \leq i \leq m .\end{cases}
$$

$\mathrm{G}(\mathrm{V}, \mathrm{E})$ is a graph obtained by merging the Dissimilar Alloys AA2024 and AA7074. Let each edge $E=\left\{a_{i j} a_{i(j+1)}: i=1,2, \ldots, m, j=1,2, \ldots, n-1\right.$ and $\left.a_{i j} a_{(i+1) j}: i=1,2, \ldots, m-1, j=1,2, \ldots, n\right\}$. Let $R_{i}$ represent the rows where $\mathrm{i}=1,2,3, \ldots, \mathrm{m}$.

Due to compression the changes in each of $\left(a_{i j}\right)$ give rise to new graph $G_{c}\left(V_{c}, E_{c}\right)$ which induces a mapping $f^{*}: V^{*} \rightarrow R^{*}$ (i.e. $b_{i j}$ represent the vertices identified by $i^{\text {th }}$ row and $j^{\text {th }}$ column, where $i=1,2, \ldots, m_{g}, j=1,2, \ldots, n_{s}$ ), where

$V_{c}=\left\{b_{i j} ; 1 \leq i \leq m_{g} ; 1 \leq j \leq n_{s}\right\}$,

$E_{c}=\left\{b_{i j} b_{i(j+1)}: 1 \leq i \leq m_{g} ; 1 \leq j \leq(n-1)_{s} \bigcup\left\{b_{i j} b_{(i+1) j}: 1 \leq i \leq\right.\right.$

$\left.(m-1)_{g} ; j=1,2, \ldots, n_{s}\right\}$

\section{Algorithm:}

\section{Before compression test:}

Input: The number of rows $m$ and columns $n$ of the graph G.

begin

$V=\left\{\left(a_{i j}\right) ; 1 \leq \mathrm{i} \leq \mathrm{m}, 1 \leq \mathrm{j} \leq \mathrm{n}\right\}$

for $\mathrm{i}=1$ to $\mathrm{m} ; \mathrm{n} \equiv 0(\bmod 2)$

\{if $(j=1)$

$$
\begin{aligned}
& \left\{f\left(a_{i j}\right)=2 i-1\right\} \\
& \text { for }(j=2 \text { to } n / 2) \\
& \left\{f\left(a_{i j}\right)=a_{m(j-1)}+2 i\right\} \\
& \quad \text { if }(j=n / 2+1) \\
& \quad\left\{f\left(a_{i j}\right)=2 i\right\} \\
& \quad \text { for }(j=n / 2+2 \text { to } n) \\
& \quad\left\{f\left(a_{i j}\right)=a_{m(j-1)}+2 i\right\} \\
& \}
\end{aligned}
$$

for $\mathrm{i}=1$ to $\mathrm{m} ; \mathrm{n} \equiv 1(\bmod 2)$

\{if $(j=1)$ 


$$
\begin{aligned}
& \left\{f\left(a_{i j}\right)=2 i-1\right\} \\
& \text { for }(j=2 \text { to }\lfloor n / 2\rfloor) \\
& \left\{f\left(a_{i j}\right)=a_{m(j-1)}+2 i\right\} \\
& \text { if }(j=\lfloor n / 2\rfloor+1) \\
& \left\{f\left(a_{i j}\right)=2 i\right\} \\
& \text { for }(j=\lfloor n / 2\rfloor+2 \text { to } n) \\
& \left\{f\left(a_{i j}\right)=a_{m(-1)}+2 i\right\}
\end{aligned}
$$

\} .

$E=\left\{a_{i j} a_{i(j+1)}: i=1,2, \ldots, m, j=1,2, \ldots, n-1\right.$ and $a_{i j} a_{(i+1) j}: i=$ $1,2, \ldots, m-1, j=1,2, \ldots, n\}$.

Output: The Graph G.

After compression test:

Input:

$E_{c}=\left\{b_{i j} b_{(j+1)}: 1 \leq i \leq m_{g} ; 1 \leq j \leq(n-1)_{s} \cup\left\{b_{i j} b_{(i+1) j}: 1 \leq i \leq\right.\right.$

$\left.(m-1)_{g} ; j=1,2, . ., n_{s}\right\}$

for $\left(i=1\right.$ to $m_{g}, j=1$ to $\left.n_{s}\right)$

\{

for $\left(h=1 \leq n_{s}\right)$

$\mathrm{e}_{\mathrm{h}}=\left(\max \left(\mathrm{b}_{\mathrm{ij}}, \mathrm{b}_{\mathrm{i}(\mathrm{j}+1)}\right) /\left(\min \left(\mathrm{b}_{\mathrm{ij}}, \mathrm{b}_{\mathrm{i}(\mathrm{j}+1)}\right)\right\}\right.$

ffor $\left(h=1 \leq n_{s}\right)$

$\mathrm{e}_{\mathrm{v}}=\left(\max \left(\mathrm{b}_{\mathrm{ij}},\left(\mathrm{b}_{(\mathrm{i}+1) \mathrm{j}}\right) /\left(\min \left(\mathrm{b}_{\mathrm{ij}}, \mathrm{b}_{(\mathrm{i}+1) \mathrm{j}}\right)\right\}\right.\right.$

\}

Output: The Graph $\mathrm{G}_{\mathrm{c}}$.

Result: If each of the elements of $e_{h}$ and $e_{v}$ is found to be distinct then the welding is stronger else not stronger.

\section{Illustration}

\section{Initial State}

Let $G=(V, E)$ where $V=\left\{a_{i j} ; 1 \leq i \leq 7,1 \leq j \leq 4\right\}$ i.e. $a_{i j}$ represent the vertices identified by $i^{\text {th }}$ row and $j^{\text {th }}$ column, and $E=\left\{a_{i j} a_{i(j+1)}: i=1,2,3,4,5,6,7, j=1,2,3\right.$ and $a_{i j}$ $\left.a_{i+1 j}: i=1,2,3,4,5,6, j=1,2,3,4\right\}$, where $k=1,2, \ldots, 24$.i.e.

$E=\left\{a_{11} a_{12}, a_{12} a_{13}, a_{13} a_{14}, a_{11} a_{21}, a_{12} a_{22}, a_{13} a_{23}, a_{14} a_{24}\right.$, $a_{21} a_{22}, a_{22} a_{23}, a_{23} a_{24}, a_{31} a_{32}, a_{32} a_{33}, a_{33} a_{34}, a_{21} a_{31}$, $a_{22} a_{32}, a_{23} a_{33}, a_{24} a_{34}, a_{41} a_{42}, a_{42} a_{43}, a_{43} a_{44}, a_{31} a_{41}, a_{32} a_{42}$ $, a_{33} a_{43}, a_{34} a_{44}, a_{41} a_{51}, a_{42} a_{52}, a_{43} a_{53}, a_{44} a_{54}, a_{51} a_{52}, a_{52} a_{53}$ , $a_{53} a_{54}, a_{51} a_{61}, a_{52} a_{62}, a_{53} a_{63}, a_{54} a_{64}, a_{61} a_{62}, a_{62} a_{63}$, $a_{63} a_{64}, a_{61} a_{71}, a_{62} a_{72}, a_{63} a_{73}, a_{64} a_{74}, a_{71} a_{72}, a_{72} a_{73}$, $\left.a_{73} a_{74}\right\}$. Let $f: V \rightarrow R^{*}$, where $R^{*}$ is a set of real numbers

$$
f\left(a_{i j}\right)= \begin{cases}2 i-1 & \text { if } j=1, i=1,2,3,4,5,6,7 \\ a_{71}+2 i & \text { if } j=2, i=1,2,3,4,5,6,7 \\ 2 i & \text { if } j=3, i=1,2,3,4,5,6,7 \\ a_{73}+2 i & \text { if } j=4, i=1,2,3,4,5,6,7 .\end{cases}
$$

Let $R_{i}$ represent the rows where $i=1,2,3,4,5,6,7$ whose entries are elements of $\left(a_{i j}\right) ; 1 \leq \mathrm{i} \leq 7,1 \leq \mathrm{j} \leq 4$ 


\section{Result}

The max/min edge Labeling (shown in Table 3 ) is computed for each edge after compression test by using $\mathrm{e}_{\mathrm{k}}=\left\{\left(\max \left(\mathrm{b}_{\mathrm{ij}}, \mathrm{b}_{\mathrm{i}(j+1)}\right) /\left(\min \left(\mathrm{b}_{\mathrm{ij}}, \mathrm{b}_{\mathrm{i}(j+1)}\right)\right\} \bigcup\left\{\left(\max \left(\mathrm{b}_{\mathrm{ij}},\left(\mathrm{b}_{(\mathrm{i}+1) \mathrm{j}}\right) /\right.\right.\right.\right.\right.$ $\left(\min \left(b_{i j}, b_{(i+1) j}\right)\right\}$

$1 \leq \mathrm{i} \leq 4,1 \leq \mathrm{j} \leq 4$.

is found to be distinct real numbers therefore the Welding is stronger.

Table 3. Computation of Max/mini of edges

\begin{tabular}{|c|l|}
\hline Edges $\left(\mathrm{e}_{\mathrm{k}}\right)^{\prime} \mathrm{s}$ & \multicolumn{1}{c|}{$\begin{array}{c}\text { Computation of Max/mini } \\
\text { of edges }\end{array}$} \\
\hline $\mathrm{e}_{1}$ & 8 \\
\hline $\mathrm{e}_{2}$ & 5.333333 \\
\hline $\mathrm{e}_{3}$ & 5.666667 \\
\hline $\mathrm{e}_{4}$ & 2 \\
\hline $\mathrm{e}_{5}$ & 1.125 \\
\hline $\mathrm{e}_{6}$ & 1.66666667 \\
\hline $\mathrm{e}_{7}$ & 1.123 \\
\hline $\mathrm{e}_{8}$ & 4.5 \\
\hline $\mathrm{e}_{9}$ & 3.6 \\
\hline $\mathrm{e}_{10}$ & 3.8 \\
\hline $\mathrm{e}_{11}$ & 2.5 \\
\hline $\mathrm{e}_{12}$ & 1.333333 \\
\hline $\mathrm{e}_{13}$ & 2.2 \\
\hline $\mathrm{e}_{14}$ & 1.375789 \\
\hline $\mathrm{e}_{15}$ & 2.4 \\
\hline $\mathrm{e}_{16}$ & 2.181818 \\
\hline $\mathrm{e}_{17}$ & 2.72727 \\
\hline $\mathrm{e}_{18}$ & 1.4287143 \\
\hline $\mathrm{e}_{19}$ & 1.14285714 \\
\hline $\mathrm{e}_{20}$ & 1.375 \\
\hline $\mathrm{e}_{21}$ & 1.4666667 \\
\hline $\mathrm{e}_{22}$ & 3 \\
\hline $\mathrm{e}_{23}$ & 2.625 \\
\hline $\mathrm{e}_{24}$ & 2.75 \\
\hline & \\
\hline & \\
\hline & \\
\hline & \\
\hline & \\
\hline & \\
\hline & \\
\hline & \\
\hline & \\
\hline & \\
\hline & \\
\hline & \\
\hline & \\
\hline
\end{tabular}

\section{Conclusion}

In a welded plate a compressive test being conducted by which it is proven that by using Max/mini edge labeling welding is stronger.

\section{References}

1. Gallian JA (2011) A dynamic survey of graph labeling. The Electronic J. of Combinatoric. 18, DS6. 